\title{
Prima e dopo la letteratura. Il veneziano e il fantasma della grammatica ${ }^{1}$
}

\author{
Riccardo Drusi \\ Piermario Vescovo
}

\begin{abstract}
La secolare storia della letteratura in veneziano non conosce una riflessione o una sistemazione grammaticale fino ad oltre la caduta dello Stato Veneto, e la stessa sistemazione di un vasto patrimonio linguistico attraverso lo strumento di un dizionario - dopo alcuni tentativi — si realizza solo con l'unità d'Italia. Questo contributo affronta brevemente la questione e propone una serie di tracce per una ricognizione del problema (soprattutto in rapporto allo scarto rispetto alla lingua letteraria e alla sua grammatica) all'interno di questa storia.
\end{abstract}

Parole chiave: Venezia, veneziano (dialetto), veneziana (letteratura), grammatica.

\section{Abstract}

Grammatical reflection or systemisation is wholly unknown in the secular history of literature in Venetian until after the downfall of the Venetian State; indeed, the very systemisation - and this somewhat tentatively — of a vast linguistic heritage through the instrument of a dictionary comes about only with the unity of Italy. This article briefly addresses the issue and puts forward a series of outlines by which to recognise more effectively the problem within this history (above all, as this relates to the very slight respect shown towards literary language and to its grammar).

Key words: Venice, Venetian (dialect), Venetian (literature), grammar.

Può apparire paradossale che alla precoce fortuna storica del veneziano non corrisponda - come succede del resto agli altri principali dialetti italiani un'altrettanto precoce sistemazione grammaticale. Rischia insomma di sorprendere che la larga attestazione documentale di questa lingua già fra Due e

1. Pur nella comune ideazione - cui va ascritta l'introduttiva sezione $0-\mathrm{i}$ paragrafi $1-2$ appartengono a Riccardo Drusi, i paragrafi 3-4 a Piermario Vescovo. Gli autori fanno parte del progetto di ricerca nazionale, cofinanziato dal MIUR, «Vocabolario dei dialetti veneti - Testi veneti dalle origini al XIX secolo», al quale aderiscono le Università di Pisa (Normale), Padova, Udine e Venezia. 
Trecento e la sua espansione non solo nei territori limitrofi alle lagune, ma - come da tempo è stato verificato ${ }^{2}$ — anche nelle aree mediterranee interessate dall'attività commerciale di Venezia, e poi la sua secolare fortuna letteraria, su ampia scala, tra il Quattro e il Settecento, non abbiano prodotto la ricerca di una norma che ratificasse su basi teoriche il consenso ormai acquisito di fatto. Si veda lo stupore di un viaggiatore francese negli anni ottanta del Settecento a proposito dell'assenza di un siffatto strumento per soddisfare le sue curiosità linguistiche:

J'ai cherché dans plusieurs villes d'Italie, une Grammaire et un Dictionnaire où je pusse m'instruire des principes de cette dialecte; il m’a été impossible de trouver nulle part, ni l'une ni l'autre; et l'on m'a assuré, dans Venise même, qu'il n'en existe pas. ${ }^{3}$

Per incontrare una prima grammatica "positiva» sembra si debba attendere il tardo, se non estremo o postumo, isolato, cauto e affatto privato esperimento di Daniele Manin, per altro solo in anni a noi prossimi riemerso dalle sue carte. Un disegno di grammatica — rilevante ma di fatto abortito — dovuto certo alla sollecitazione del Dizionario del dialetto veneziano di Giuseppe Boerio (prima edizione, 1829; seconda edizione "aumentata e corretta», 1856), nella cui redazione Manin fu fattivamente coinvolto dall'autore. ${ }^{4}$

Benché tutta ispirata a una volontà di testimonianza e di reazione nel presente - e da parte di una personalità politica (su cui gli storici discutono ancora) che tenterà un anacronistico rilancio dello stato veneziano nella gloriosa insurrezione antiaustriaca del 1848-49 — la piccola Grammatica del Manin, insieme alla più consistente fatica del Boerio, finiscono col rappresentare un momento di passaggio in cui, come è stato scritto, «l'erudita pietas locale prendeva in consegna un secolare patrimonio di lingua e letteratura e lo trasmetteva integro alla filologia dell'Italia unita». ${ }^{5}$

2. Cfr. G. FolENA, «Introduzione al veneziano de là da mar», in ID., Culture e lingue nel Veneto medievale, Padova: Editoriale Programma, 1990, p. 227-267, con bibliografia.

3. Il passo è citato in A.L. MomOgLIANO LePSCHY, "Un "rudiment vénitien" del Settecento", in Atti dell'Istituto Veneto di Scienze Lettere ed Arti, Classe di scienze morali e lettere, CXXII, 1963-1964, p. 453-481: cfr. p. 476. Il viaggiatore francese - che il saggio prova ad identificare - si dedica ad alcune annotazioni sul dialetto veneziano, tuttavia di scarso, se non nullo, interesse linguistico.

4. La Grammatica del dialetto veneziano è stata esumata dal fondo Manin-Pellegrini della Biblioteca del Civico Museo Correr di Venezia e pubblicata da Angela Caracciolo Aricò in "Quaderni Veneti», n. 3, 1986, p. 11-39 (col corredo di una nota linguistica di Manlio Cortelazzo); della stessa studiosa, sul fronte del coinvolgimento in rapporto al Dizionario del Boerio, cfr. A. Caracciolo ArICò (a cura di), Daniele Manin editore. Carteggio Daniele Manin - Giuseppe Boerio, Roma: Bulzoni, 1984.

5. A. STUSSI, «La letteratura in dialetto nel Veneto», in ID., Lingua, dialetto, letteratura, Torino: Einaudi, 1993, p. 64-106, p. 92. 
1.

Per cogliere il clima in cui l'opera del Boerio - e, accanto ad essa, su piano ben diverso, il breve tentativo di descrizione fonetico-morfologica del Manin - si inserisce, basti rammentare l'esagerazione della retorica in domande come queste, con le quali il lessicografo apriva la sua fatica:

Qual altro in fatti de' dialetti italiani si mostrò con più facile riuscita rivale nella forza e nelle grazie all'antica sua madre? Grave e fecondo persuase nella tribuna de' comizi Veneti, e si ricordano con onore nella storia, tra mille altri, i nomi illustri degli arringatori patrizii [...].

Non senza abuso della storia, perché la filza di «arringatori patrizii» che segue comprende nomi la cui pratica oratoria è documentata nella sola lingua di Roma o, eventualmente, nel toscano letterario della classicità volgare successiva alla codificazione del Bembo.

Ma l'intento del Dizionario, che era poi quello di mostrare come e quanto un lessico regionale potesse contribuire all'auspicata lingua nazionale, ${ }^{6}$ era nobile a sufficienza per tollerare la forzatura, né le fortune delle scienze linguistiche in Italia erano tali da consentire un argine a questo o ad altri entusiasmi patriottici.

Colpisce invece che anche dopo il Boerio, e nonostante i progressi intervenuti nei pertinenti ambiti disciplinari, le esagerazioni non venissero meno: è noto il pregiudizio di Giusto Grion e Adolfo Bartoli sulla totale «veneticità» (se non persino venezianità) del manoscritto Saibante-Hamilton 390, l'antica silloge di testi morali che, come dimostrò l'Ascoli, tramanda nel suo complesso una facies linguistica genericamente settentrionale, comunque priva di localismi marcati. Ancora nel 1891 Enrico Bertanza e Vittorio Lazzarini licenziavano per le stampe una prima e pionieristica raccolta di documenti volgari intitolandola perentoriamente Il dialetto veneziano fino alla morte di Dante: ${ }^{7}$ nel regesto vennero ammessi (sia pure con prese di distanza da parte dei curatori, e spesso sotto la formula anodina della notizia bibliografica) reperti dubbi o chiaramente estranei al contesto lagunare, ma utili — ancor prima che all'esaurimento della bibliografia pregressa - alla tesi di un veneziano come lingua della «miglior

6. L'idea, palesemente informata alle dottrine di Melchiorre Cesarotti, meglio appariva nella prefazione, rimasta inutilizzata, che per il Dizionario aveva compilato il già ricordato Daniele Manin, del Boerio corrispondente e interlocutore elettivo in materia di lingua: «Questa Italia, la cui lingua deve servire a spiegare le idee di tutti i suoi abitanti, chiude in suo seno molte province, le quali tutte sia per diversità di origine, sia per varietà di politiche vicende hanno un dialetto particolare. Sarà dunque onesto e profittevole che queste province, siccome parti della comune patria, rechino in tributo quelle voci e quelle frasi di cui abbisogna la comune lingua; in A. CARACCIOlO ARICÒ (a cura di), Daniele Manin editore. Carteggio D. Manin - G. Boerio, cit., p. 215; cfr. anche ID., "Daniele Manin editore», Quaderni Veneti, n. 31-32, 2000, p. 199-209: p. 204.

7. E. BERTANZA e V. LAZZARINI, Il dialetto veneziano fino alla morte di Dante Alighieri 1321, Venezia: Editrice di M. S. fra Compositori, 1891 (una ristampa anastatica ha procurato, nel 1974, l'editore Forni di Bologna). 
parte» della popolazione, preservatasi ab immemorabili fino alla caduta della Repubblica Veneta e simbolo eloquente della sua indipendenza politica.

Ora se si considera che Venezia, com'è detto nella famosa lettera di Cassiodoro ai Tribuni marittimi, trasse le sue origini precisamente dalla miglior parte di questi veneti - i quali al ruinare della potenza di Roma e nell'imperversare delle irruzioni barbariche cercarono rifugio fra queste lagune, e qui, per ben quattordici secoli, non videro né patirono, etnicamente parlando, straniere pervasioni - come non si potrà affermare, che il vernacolo di Venezia è da considerarsi ancora tra i più schietti e genuini avanzi dell'antichissima civiltà d'Italia? [p. VIII].

Ovvio che ciascuna di queste posizioni aveva qualche fondamento nei fatti. La possibilità di dipingere il veneziano di volta in volta come veicolo 'ufficiale' dell'amministrazione della Serenissima, come lingua formale dei più antichi statuti insulari, come idioma esclusivo del foro veneto richiede condizioni sostanziali: e queste certo vi furono, anche se (e si torna con questo al problema sollevato all'inizio) mai ebbero a verificarsi in misura tanto estrema da sottintendere quella precisa consapevolezza nell'uso di un idioma che, sola, consente e legittima la sua cristallizzazione in regole di grammatica. L'eccellente lavoro di Lorenzo Tomasin sul volgare nella prassi giuridica Repubblica Veneta ha confermato che di una ufficialità del veneziano si può parlare soltanto a partire dal tardo Trecento, e con la significativa restrizione, allora e poi, entro il circuito dell'oralità, le scritture continuando a prediligere il latino e, dal XV secolo, un italiano sovraregionale. ${ }^{8}$ Come dire — esasperando i termini della questione che se il dibattito politico e giudiziario, importante ma per sua natura effimero, poteva valersi (con molti accorgimenti) della parlata quotidiana, la formulazione scritta della legge aveva invece esigenze di stabilità, durata e diffusione cui solo l'universale latino o una 'lingua comune' potevano corrispondere.?

Dunque, se di consapevolezza linguistica si può parlare, facile definirla piuttosto come un sentimento istintivo nei confronti dell'idioma usuale: quello che — si può facilmente presumere - avrà permesso a un veneziano del Duecento di riconoscere per veneziano o meno il suo interlocutore. Proprio perché istintivo, questo sentimento aveva tuttavia scarse probabilità di convertirsi in una definizione linguistica positiva. Un pieno statuto linguistico poteva, all'epoca, competere unicamente al latino, cioè a quello che appariva come il modulo espressivo universale, incorruttibile, svincolato - a differenza dei volgari - dalle vicissutidini della storia e, in breve, il solo dotato di una grammatica: anzi, esso stesso grammatica per definizione. E se proprio la struttura grammaticale costituiva il principio di individuazione del latino, ecco che

8. Cfr. L. TOMASIN, Il volgare e la legge. Storia linguistica del diritto veneziano (secoli XIII-XVIII), Padova: Esedra editrice, 2001, p. 34-35.

9. Si veda al riguardo il puntuale intervento di M. CORTELAZZO, «Il veneziano, lingua ufficiale della Repubblica?», in M. CorTELAZzo (a cura di), Guida ai dialetti veneti, IV, Padova: Cleup, 1982, p. 59-73, in particolare p. 73. 
per contrario ogni possibile codificazione formale era preclusa alle lingue correnti già in linea di principio, con, dunque, un ulteriore pregiudizio alla possibilità di definirne specificità e caratteri. Dando circolarità al ragionamento, il volgare (qualsiasi volgare) non aveva identità precisa perché non aveva grammatica, e non aveva grammatica perché, ovviamente, non poteva competere con il latino sotto alcun aspetto.

Con questa - come dire? - sensibilità attenuata verso la lingua usuale, si comprende bene come un impiego formale del volgare, per il fatto stesso di sconfinare dalla dimensione meramente istintiva dell'uso quotidiano verso zone di applicazione riflessiva e ponderata (quelle sostanzialmente rivendicate dal latino), richiedesse operazioni di adattamento compromissorie e nettamente snaturanti: e, per tornare alla questione più sopra enunciata, i volgari non tanto muovevano da una precisa identità per assumere nuove funzioni, quanto piuttosto acquisivano un'effimera identità dalla funzione via via ricoperta. La circostanza è stata evidenziata dagli addetti ai lavori con il concetto di scripta, formulato a rimarcare l'alterità della dimensione grafica del volgare (specie d'ambito documentale) dalla sua base orale e dell'uso generale. Di questa dimensione sono sintomatici i fenomeni di generalizzazione che si segnalavano per i testi del codice Saibante e che, rispetto ai documenti notarili veneziani, sono stati ottimamente evidenziati da Alfredo Stussi nei testi da lui editi e analizzati. Per soprammercato, la Venezia medievale conosceva ulteriori ostacoli alla crescita di un'idea forte di lingua. Si tratta di mancanze e di condizioni svantaggiose. Come ha notato ancora una volta Stussi, ${ }^{10}$ Venezia, che dilata i propri mercati fino al Levante, è priva in patria di qual si sia organismo suscettibile di divenire polo gravitazionale della lingua: manca una corte signorile a promuovere la pratica letteraria in volgare, e manca uno studium universitario che incroci esperienze culturali e solleciti la sperimentazione (la sola università «veneziana» sarà quella di Padova, frutto delle conquiste quattrocentesche in terraferma: per calcolo prudenziale, i governanti non l'avvicinarono mai alla capitale). Quanto agli svantaggi, è significativa la presenza a Venezia di una classe notarile costituita esclusivamente da religiosi, meno propensi dei loro omologhi laici, siciliani e toscani, a rivolgere l'attenzione al volgare come possibile lingua letteraria; ma è prima di tutto la vocazione mercantile di Venezia a causare ritardi alla crescita linguistica, perché proprio l'apertura massima verso l'esterno e, insieme, il quotidiano confronto con una pluralità di idiomi inibiscono decisamente la formazione d'un baricentro linguistico locale. Prove della tolleranza verso le parlate dei forestieri che affollavano i fondaci veneziani si hanno nella scelta, da parte di Martino da Canal, del francese come lingua per redigere la sua cronaca duecentesca, ${ }^{11}$ e nel successo precoce

10. «La letteratura in dialetto nel Veneto», cit., p. 65-66.

11. «[...] lengue franceise cort parmi le monde et est la plus delitable a lire et a oïr que nule autre», MARTIN DA CANAL, Les estoires de Venise, I, 5 (a cura di A. LiMENTANI, Firenze: Olschki, 1973). Cfr. anche F. BRUGNOLO, «I toscani nel Veneto e le cerchie toscaneggianti», in AA. VV., Storia della Cultura veneta, 2, Il Trecento, Vicenza: Neri Pozza, 1976, p. 369-439, p. 383-384. 
e travolgente della lirica toscana fra le lagune durante il Trecento; e se non fosse che così facendo si distorcerebbero reali presupposti e si ignorerebbero comprovate cause culturali, verrebbe insomma da integrare a questa serie anche la venezianità del Bembo, che grammatico fu non della propria, ma della lingua di Firenze.

L'aver ricordato la cinquecentesca grammatica bembiana fa riflettere sul fatto che solo dopo di essa, e dunque solo dopo la fissazione d'una lingua per la prima volta davvero «italiana», si incontra quella letteratura riflessa, parodica del canone sancito dalla norma, che trova nei dialetti (e il veneziano primeggia fra gli altri) il mezzo espressivo d'elezione. $\grave{E}$, anzi, questa la condizione che permette di parlare davvero di dialetti e non più di lingue locali: la realizzazione d'un sistema gerarchico anche nell'ambito del volgare segna infatti demarcazioni precise fra ciò che nei testi letterari è linguisticamente ammissibile e ciò che non lo è più, e tutelando il fiorentino trecentesco, ormai consacrato a lingua della letteratura più elevata, entro confini segnati con estrema precisione, delimita spontaneamente ma con altrettanta nettezza anche le aree dialettali destinate a rimanere esterne. Proprio perché definitivamente estromessi dagli spazi dell'aulicità, i dialetti smettono ogni paludamento e, dai registri dimessi - parodici, appunto — che sono stati loro attribuiti, possono affacciarsi per la prima volta nella loro spontanea naturalezza. Come un opportuno catalizzatore fa precipitare in cristalli solidi l'elemento precedentemente dissolto nel liquido, così la grammatica bembiana ebbe la facoltà di separare nelle scritture la lingua eletta, quella letteraria, dagli altri moduli idiomatici italiani, fino ad allora fluttuanti in mescidanze linguistiche che ne offuscavano la fisionomia. Per quanto riguarda il veneziano, esiti della sua condensazione per effetto della grammatica del Bembo si misurano bene nella Ortografia delle voci della lingua nostra o vero Dittionario volgare e latino che Francesco Sansovino - il poligrafo ed editore figlio del più noto Jacopo: toscano di nascita, ma veneziano d'adozione stampa a Venezia nel 1568. In questo lessico della lingua letteraria — significativamente dichiarata, a titolo, nostra - ai vocaboli toscani vengono frequentemente associati sinonimi veneziani, con un procedimento che risponde anzitutto alla pratica esigenza di comprensibilità dei lemmi ma che, insieme, fissa per iscritto molte voci vernacole altrimenti prive di attestazione:

Asse per s dop(pia)] Assi, Tabula, Tavola di legno. Tola dicono i Vinitiani. Bando] Praeconium. La grida dicono i Vinitiani [...]

Basilico per $s$ e $c$ semp(lice)] Ocimum, [...] Basigò si dice a Venetia.

Bavero per $r \operatorname{semp}($ lice)] [...] Lista di panno o di seta che va attorno al collo.

Collaro lo dicono i Vinitiani.

Calcina $]$ calx, quam sit, cal[c]e. Malta si dice a Venetia per muratori.

Cannone] Tubus, doccia, gorna a Venetia.

Cantina per $n$ semp(lice)] Cellaria, volta, canova dicono a Venetia [...].

Gheroni per $r$ e $n$ semp(lice)] quelle giunte delle vesti, che si chiamano ghedi a Venetia, o scuetti.

Ruggine per $g$ dop(pia) e $n$ sem(plice)] Ferrugo, rubigo, ruzzene dicono a Venetia, nemica del ferro [...]. 
Grembiale per $l$ semplice] cincticulus, gremmiale, traversa si dice a Venetia $[\ldots]^{12}$

A verificarsi è proprio la presa di coscienza della lingua locale nella sua interezza: quella consapevolezza linguistica cui si faceva cenno come al requisito essenziale di ogni grammatica. La letteratura cinquecentesca in veneziano non si limita infatti, né può a limitarsi, a registrare servilmente il parlato. La funzione espressivistica cui si assolve scrivendo in dialetto comporta infatti un rapporto dinamico con l'elemento linguistico, che viene vagliato e selezionato accuratamente in rapporto agli obiettivi stilistici prefissati. L'operazione di resa letteraria, come ogni azione che miri a uno scopo, non procede se non sulle basi di un metodo, per quanto embrionale e limitato esso sia: con il che viene da postulare - provocatoriamente, ma fino a un certo punto - una sorta di grammatica implicita, un vaglio invisibile ma tramato di solide categorie lessicali, morfologiche, sintattiche e stilistiche, di cui l'autore si serve per classificare i materiali grezzi e discernere i più rispondenti alla bisogna. Non si tratta più di modellare la lingua su elementi allotri, come era avvenuto nella prima stagione del veneziano scritto, fra Due e Trecento. Quello che si ricerca è precisamente il contrario del volgare depurato, sovraregionale, coerente per quanto possibile con l'onnipresente modello di riferimento, il latino, e con altri volgari limitrofi cui tendevano i duecenteschi Proverbia que dicuntur super natura feminarum (se sono veramente veneziani: la difficoltà di collocarli esprime bene l'alto tenore di tratti interlinguistici di questo testo), i coevi volgarizzamenti del Panfilo ${ }^{13}$ e del Cato, ${ }^{14}$ e poi i trecenteschi De regimine rectoris di Paolino Minorita, ${ }^{15}$ l'Apollonio di Tiro, ${ }^{16}$ testi agiografici come il Santo Stady di Franceschino Grioni ${ }^{17}$ e l'anonima Legenda de misier Sento Alban ${ }^{18}$ con gli altri monumenti della letteratura veneziana medievale; nemmeno si aspira più alla lingua improntata ai formalismi giuridici degli atti studiati da Stussi, piatta e uniforme, e solo casualmente più vivace se il testo non passava per le mani del notaio (è questo il caso del testamento, codicillo testamentario e conti autografi di Geremia Ghisi, dello scorcio del Duecento, nei quali si ha ben saldo il dileguo delle dentali intervocaliche - contraa, moo per contrada, modo -

12. Si cita, modernizzando la grafia e sciogliendo le abbreviazioni, da C. MARAzzINI, «Un editore del Cinquecento tra Bembo e il parlar popolare: F. Sansovino ed il vocabolario», Studi di lessicografia italiana, n. 5, 1983, p. 193-208, p. 204-205.

13. Il Panfilo veneziano, edizione critica con introduzione e glossario a cura di H. HALLER, Firenze: Olschki, 1982.

14. Edito dal Tobler, «Die altvenezianische Übersetzung der Sprüche des Dyonysius Cato", Abhandlungen der k. Preussischen Akademie der Wissenschaft zu Berlin, 1883, p. 1-87.

15. Se ne veda l'edizione a cura del Mussafia, Tendler e Vieusseux, Vienna-Firenze, 1868.

16. La storia di Apollonio di Tiro, versione tosco veneziana della prima metà del sec. XIV, edita da C. Salvioni, Nozze Solerti - Saggini, Bellinzona: Salvioni, 1889.

17. A. Monteverdi, «La leggenda di Santo Stady di Franceschino Grioni», Studi Romanzi, n. 20, 1930, p. 1-199.

18. "Legenda de misier Sento Alban". Volgarizzamento veneziano in prosa del XIV secolo, edizione critica a cura di E. BURGIO, Venezia: Marsilio, 1995. 
viceversa conservate nei testi letterari. ${ }^{19}$ Presenta andao, desprexiao anche un documento del 1281 pubblicato da Belloni e Pozza: ${ }^{20}$ trattandosi di una scrittura privata, ${ }^{21}$ anche per essa si potrà presumere una minore sorvegliatezza formale). Per i cinquecentisti, il tono giocoso e parodico convenzionalmente vincolato alla letteratura in dialetto obbliga alla cernita di quanto più si distacca dalla norma linguistica «ufficiale»: dunque, in opposizione al fiorentino letterario, degli elementi localmente più connotati, dei vocaboli più caratteristici, delle forme idiomatiche di più stretta pertinenza. Il lessico del Sansovino poco sopra citato mostra quanta e quale fosse la disponibilità di tali elementi per un professionista delle lettere di medio Cinquecento, anche se di origine non veneziana. Fra quanti, quali Calmo, Caravia, il Burchiella, Maffio Venier, nelle lagune erano nati, più agevole riusciva impigliare voci, morfemi, costrutti e locuzioni fra le maglie di un setaccio che, per corrispondere a tale scopo, doveva appunto essere tramato anche di categorie grammaticali. Era, in fondo, il medesimo setaccio impiegato nelle soluzioni di coiné che si son viste caratterizzare i testi veneziani due e trecenteschi; ma impiegato per così dire al contrario, cioè al diverso fine di lasciar fluire la parte sierosa - la diafana sostanza comune ad altri sistemi linguistici —, e di raccogliere invece le scorie degli idiotismi.

\section{2.}

Testi «riflessi» in veneziano si hanno peraltro già in epoca anteriore al Cinquecento, e di questi si cercherà di dar conto come di altrettante emersioni della "grammatica implicita» cui si accennava. La casistica, compresa fra il Tre e il Quattrocento, è ovviamente sporadica a causa dell'assenza dell'unificazione linguistica operata, come si è visto, dal Bembo, ma è di particolare interesse in quanto rappresentata essenzialmente da scriventi alloglotti, e come tali non sospettabili di una mimesi spontanea della lingua. L'appropriazione di un mezzo espressivo altrui comporta, anzi, che ciascuno di loro operasse secondo schemi linguistici razionalmente dedotti: che procedesse, in sostanza, ad astrazioni formali di chiara impronta grammaticale, analizzando la massa bruta dell'idioma da riprodurre e raggruppandone lemmi, forme, costrutti in comparti categoriali utili quanto più si rendevano di immediata riconoscibilità. Scontatamente — ma non è sconveniente ricordarlo — a potenzialità grammaticali siffatte mancò qualsiasi eventualità di generalizzazione, sia per il carattere dimesso dei testi (fondamentalmente caricaturali, legati al genere del vituperium contro i titolari della lingua usata: «riflessi», appunto; o, in un paio di casi, pertinenti alla sfera pratica della contabilità mercantile e del dispaccio

19. Cfr. A. STussi, Testi veneziani del Duecento e dei primi del Trecento, Pisa: Nistri-Lischi, 1965, n. 4, 5, 6 e commento linguistico, p. LVII-LVIII.

20. G. Belloni; M. PozZA, Sei testi veneti antichi, Roma: Jouvence, 1987. Il testo, che si legge a p. 54, è una "demandaxon" (petizione) alla Signoria veneziana in una controversia di materia patrimoniale. Per i tratti qui evidenziati, si veda la Nota sulla lingua, p. 22.

21. Cfr. ibid., p. 46. 
diplomatico), sia per l'autonomia in cui gli scriventi operarono, senza che mai — e diversamente da quanto avverrà appunto nel Cinquecento — i rispettivi prodotti si contagiassero a formare una tradizione: quanto dire che se si accetta, per essi, di parlare d'un sottofondo grammaticale, questo fu comunque fenomeno individuale, mai affrancato da istanze peculiari e, come tale, invariabilmente sterile rispetto alla conquista d'una sensibilità linguistica comune.

\section{1}

Non si può non cominciare da Dante, che catalogando nel De vulgari eloquentia (I, XIV $)^{22}$ i linguaggi italiani non rispondenti al suo ideale di volgare letterario cita, a esempio del veneziano, quello che pare un verso (endecasillabo tronco?) di un più esteso componimento: "Per le plaghe di Dio tu no verras»; ovvero, secondo la ricostruzione che Stussi ottiene adeguando la tradizione testuale a plausibili coordinate fonetiche e paleografiche, "Per le plage de Dio tu no veras». ${ }^{23}$

Ad onta della brevità del campione, i «segni particolari» della lingua di Venezia sono abbondantemente rappresentati e, corrispondendo a quanto oggi riesumato dai documenti coevi, ribadiscono le cognizioni di causa alla base della scelta dantesca: la conservazione del nesso PL-, la -e protonica di de, in contrasto al toscano di; soprattutto, l'uscita in $-s$ per la seconda persona singolare in veras, tratto che separa il veneziano antico tanto dal toscano che dagli altri dialetti veneti, nonché da una varietà essa stessa pertinente al bacino lagunare qual era il dialetto di Lio Mazor.

\section{2 .}

Fosse anche isolato, il luogo del De vulgari eloquentia sarebbe fondamentale per capire su quali basi un orecchio genericamente italiano cogliesse, nel primo Trecento, la venezianità di un ipotetico interlocutore; ma che Dante avesse scelto per il meglio è corroborato, come si anticipava, dai numerosi altri esempi che, autonomamente dal suo trattatello, stilizzano il veneziano su moduli affini se non coincidenti.

È il caso (per osservare un ordinamento cronologico) del sonetto designato come venetus nella tenzone tridialettale, in padovano, trevisano e, appunto, veneziano del trecentesco canzoniere Colombino del trevigiano Niccolò de' Rossi. Importa ribadire che la tenzone è, nel suo insieme, il «Primo esempio in assoluto [...] di una modalità letteraria destinata a grande fortuna, quella del "dialetto come genere e come registro" ", ${ }^{24}$ e che — per quanto più

22. Si cita dall'edizione curata da P. V. Mengaldo, Padova: Antenore, 1968, p. 24.

23. Cfr. A. STUSSI, «Il dialetto veneziano al tempo di Dante», in Dante e la cultura veneta. Atti del Convegno di Studi organizzato dalla Fondazione Cini, a cura di V. BRANCA E G. PADOAN, Firenze: Olschki, 1967, p. 109-115: p. 110-111.

24. Così F. BRUgnolo, «La tenzone tridialettale del Canzoniere Colombino di Nicolò de’ Rossi. Appunti di lettura», Quaderni Veneti, n. 3, 1986, p. 41-83, p. 41. La parte virgolettata, come avverte l'autore, è citazione da C. SEGRE, La tradizione macaronica da Folengo a Gadda (e oltre). 
davvicino ci riguarda - giusto il suo plurilinguismo garantisce la consapevole stilizzazione di ciascuno dei tre dialetti, onde garantirne la riconoscibilità: quanto basta per non preoccuparsi se l'autore sia l'effettivamente veneziano "çanin» che trapela dalle repliche padovana e trevisana (forse il Giovanni Quirini imitatore di Dante a Venezia), ovvero lo stesso Niccolò de' Rossi (cui andrebbe allora ascritto l'intero trittico). ${ }^{25}$

La contingenza storica adombrata dalla tenzone parrebbe la guerra veneziana per Ferrara del $1308:^{26}$ a fronte della sospetta neutralità dei Padovani, il veneziano "çanin" rimprovera uno di loro, Guercio (Verço) da Montesanto, aggiungendo per soprammercato l'accusa di disfattismo (sarà tema ricorrente, come nota Brugnolo, anche nella frottola veneziana di Francesco di Vannozzo): segue la replica di Guercio, e un intervento super partes del trevigiano Liberale da San Pelagio, con il che il confronto si chiude. Il senso, riassume Furio Brugnolo, benemerito studioso del trittico, ${ }^{27}$ è che il padovano Guercio pratica le sedi politiche (Rialto, dove stavano molte magistrature veneziane) per diffondere «con sicumera previsioni e pareri sull'andamento e la gestione della guerra; e non devono essere certo previsioni favorevoli ai veneziani $[. .$.$] . È insomma un astrologo che predice sventura [...], un disfat-$ tista». 28

L'apostrofe diretta all'interlocutore è strategica per largheggiare nelle seconde persone singolari, la cui uscita in -s si è vista costituire un perfetto blasone di venezianità e che qui la dislocazione in rima rende affatto appariscente: montis — «sali», quindi «ti affatichi»: nell" «attingere, innalzare acqua con la coclea», (mi discosto un poco dall'interpretazione corrente) —, t'afrontis — «ti imbatti (in noi)» —, contis — «racconti» — t'apontis — «ti vanti» —; e ancora (fuori di rima) pos («puoi»), sis («sei», e «sia», v. 10), averàs («avrai»), seras («sarai»). L'area del morfema, osserva la Corti, ${ }^{29}$ è qui decisamente più larga che in testi letterari coevi: "per esempio nell'Apoll[onio] di Tiro [...] la -s risulta di norma nelle forme ossitone, facoltativa nelle parossitone». Nei testi pubblicati da Stussi si incontrano il concordante toponimo Ferera, in un documento del $1253,{ }^{30}$

25. Osserva Brugnolo, «I toscani nel Veneto e le cerchie toscaneggianti», cit., p. 410, n. 146: "In realtà la tenzone (che sembra proporsi come fine primario un'artificiale vistosa caricatura dei tre dialetti utilizzati e che dunque potrebbe essere fittizia) meglio s'inquadrerebbe nella tendenza allo sperimentalismo linguistico e all'espressionismo d'origine vernacolare che distingue de' Rossi».

26. Cfr. M. CORTI, «Una tenzone poetica del sec. XIV in veneziano, padovano e trevisano», Lettere italiane, n. 18, 1966, p. 138-151 (poi anche in Dante e la cultura veneta, cit., p. 129142).

27. Cfr. F. Brugnolo, «Per il testo della tenzone veneta del canzoniere Colombino di Nicolò de' Rossi», in Scritti linguistici in onore di Giovan Battista Pellegrini, Pisa: Pacini, 1983, voll. 2: I, p. 371-380; ID. "La tenzone tridialettale...», cit.; ID., «Ritornando sulla canzone di Auliver e altre liriche dell'età caminese. Precisazioni e proposte», Quaderni Veneti, n. 24, 1996 , p. 9-25, p.17-18.

28. BRUGNOLO, «La tenzone tridialettale...», cit., p. 47-48.

29. "Una tenzone poetica...", cit., p. 145.

30. Cfr. STUSsi, Testi veneziani, cit., p. 3; CORTI, «Una tenzone poetica...», cit., p. 144-145. 
e, con oscillazioni, il passaggio di AL + dentale a ol qui offerto da olto («alto»), Riolto («Rialto»), mesolto («misalto»: "carne di porco salata»), oltri («altri»). ${ }^{31}$ Il tratto, come vedremo, emergerà anche successivamente nell'uso letterario "riflesso", finendo, nel Cinquecento, per screziare l'arcaica lingua degli Epitaphii de molimenti antighi di Andrea Calmo. ${ }^{32}$ Anche Verço, dove $v$-sta per il toscano gu-, è da intendersi come localismo, posto che analogo passaggio si riscontra nel veneziano Zibaldone da Canal, quaderno mercantile della metà del Trecento; ${ }^{33}$ altrettanto dicasi per ficio, forma aferetica per «officio», "carica istituzionale», presente esso pure nei Testi veneziani dello Stussi.

La struttura metrica esigua permette di riferire il sonetto per intero: si segue il testo approntato da Brugnolo nell'ultimo dei suoi interventi al riguardo.

\section{Venetus}

Verço, co' tu sis struolego che montis urir aqua cum verigola ad olto!

Pesse tristo, mo' co no' < tu > t'afrontis

(e certo cusì fas tu en Riolto)

mo' stas tu coy signori, e sì contis

che 'l dose col conseio è stado molto,

e che tanto vadagnis, se t'apontis,

che pos mançar folege e mesolto.

Bestia bestia, co' < tu > sis enganado!

Vèstite ad oro e sis conparisente,

e và cum gl'oltri a l'oste de Ferera:

averàs ficio e seràs meritado.

Or oldi: no ti sgumentar nïente, cha, par Dïo, nu averemo la tera.

\section{3}

Definita dal moderno editore «curiosa testimonianza di dialetto veneziano in bocca toscana», ${ }^{34}$ la serie di appunti contabili che il mercante d'origine pisana Pignol Zucchello redasse a Venezia, divenuta sua stabile base commerciale, nel

31. Cfr. STUSSI, Testi veneziani, cit., p. XLVI-XLVII, e CORTI, "Una tenzone poetica...», cit., p. 144.

32. Cfr. A. Calmo, Le bizzarre, faconde et ingegnose rime pescatorie, testo critico e commento a cura di G. BELLONI, Venezia: Marsilio, 2003. Nell'epigrafia fittizia del Calmo la serie si presenta sotto le specie olto, Riolto (epitaffio XVI) e oltro (ep. XXX): si veda anche il glossario allestito da Belloni in appendice all'edizione.

33. Cfr. Zibaldone Da Canal. Manoscritto mercantile del sec. XIV, a cura di A. STUSSI, con studi di F. C. Lane, TH. Marston, O. Ore, Venezia: Comitato per la pubblicazione delle fonti relative alla Storia di Venezia, 1967: Note introduttive, p. XIII, n. 10.

34. Lettere di mercanti a Pignol Zucchello (1336-1350), a cura di R. Morozzo Della RocCA, Venezia: Comitato per la pubblicazione delle fonti relative alla Storia di Venezia, 1957, p. VII; il testo alle p. 71-2. 
1347, offre un colore decisamente più sbiadito che nelle testimonianze testé considerate, e l'interferenza del sistema d'origine affiora talvolta, come ad es. nella preposizione di (il veneziano predilige de). La scialbatura non è però tale da obliterare l'intenzione del menante di aderire al sistema ospite, riproducendone (con alterna efficacia) gli elementi di maggior convenienza. Così, oltre a generici tratti grafico-fonetici «settentrionali», quali la lenizione della labiale - $p$ - in avril e della dentale intervocalica - $t$ - in dado e l'affricata dentale in luogo della palatale toscana (faço, Franzescho), si osserva $v$ - per il toscano gu(varda), che si è visto peculiare alla imitazione veneziana del sonetto testé esaminato. Nel vocalismo si osservano il dittongamento in dié (<DEBET) e il passaggio ARI(US) > er in deneri, fenomeni presenti (specie il secondo) nella scripta veneziana; ${ }^{35}$ anche in Moriçio si ha un passaggio AU > -o- non insolito (ancorché qui sia in protonia) nei testi due-trecenteschi (si veda, per contrasto, la grafia latineggiante Maurocenus per 'Morosini', cognome di famiglia patrizia, nei Testi veneziani di Stussi: commento linguistico, p. XLVI). L'apertura di $e$ protonica in a di fanestra ripropone identico campione di un testo del 1305. ${ }^{36}$ Nella morfologia, il pronome personale soggetto di prima persona singolare $m i$, con poca concorrenza, segna un netto stacco dalla scripta documentale coeva - dove prevale eo di diretta derivazione latina - a tutto vantaggio di un tratto spiccatamente usuale.

\section{7, dì.vI. di avril.}

Memoria faço mi Pignol Zuchello chomo io sì ò mandado a pagar en Handia a ser Franzescho Bartollamei per mi soldi .IIII. de' grosso.

Li qual deneri die' aver per mio nome Marcho Ramella fio di Felipo Ramella e a salvameli a mia posta.

Anchora ò dado mi Pignol si mi' deneri propi a ser Felipo Ramella di Santo Moriçio ducati .LVIII. d'oro, li qual deneri ello me die' a mandar en Chandia a so' fio Marcho Ramella.

Sì che llo dito Marcho avirà del mio duchati .C. d'oro.

Li qual deneri mi Pignol sì ò mandadi a pro e dano de Vanino e Fazino e de mi Pignol.

1347, dì .vI. di avril.

Memoria faço chomo mi pignol Zuchello die' dar a frar Paxino di Noara lire .IIII. soldi .IIII. di grossi quando io saverò che Franzescho Bartolamei averà dado a Marcho Ramella per mi libre .IIII. soldi .IIII. di grossi en Handia.

Memoria faço homo ser Franzeschin da Halle me die' dar soldi .XX. di grossi delle masari' ch'è en lla staço' e volta che sta, sta mo Zane, e no' fome rasonade a mi tanto.

Dieme dar per uno charatelio di vino soldi .XI. de grossi.

Memoria faço homo Bortolamio favro di Sant'Aponal à a chasa soa una mia fanestra granda enferada la qual fo della fanestra che varda sovra la mia chorte. [...]. lo Zafior

lle sene. 
La chaxa. [...]

Ave a 'sto dì ch'en Iachomello Habriel libre .IIII. soldi .III. grossi. v l'avanço ave di borsa. ${ }^{37}$

\section{4 .}

La guerra fra Venezia e Genova che, cominciata nel 1378, vede i genovesi invadere Chioggia e minacciare gli avversari in casa propria, conosce una svolta decisiva nel luglio 1380, con la riconquista di lembi meridionali della Laguna (il Castello delle Bebbe) e il contrattacco veneziano che riuscirà infine vittorioso. La circostanza viene presa a spunto dal rimatore (di norma in lingua toscana) Francesco di Vannozzo, padovano di nascita $(1340 \mathrm{ca}$.) ma di stirpe aretina, per allestire i versi di una lunga frottola in veneziano. Qui l'imitazione linguistica serve davvero a restituire il colore locale, posto che dall'eco bellica iniziale presto si passa alla celebrazione, nel centralissimo campo di San Polo, del matrimonio fra Affenido Malipiero e Rebosa Moro, rampolli di case patrizie rivali. Collocabile dunque alle origini del genere del «mariazo», che ampio sviluppo avrà nei due secoli seguenti, il testo sfuma la parvenza conciliativa del suo contenuto in sottili allusioni alla rissosità dei veneziani (oltre al contrasto fra le famiglie Malipiero e Moro, la cerimonia stessa è turbata dai ceffoni che il novello sposo allunga, per futili motivi, alla consorte): forse un elemento caricaturale ormai passato a stereotipo, se si ritorna alle bellicose profferte del sonetto di çanin più sopra considerato, e comunque comprensibile in un autore di Padova, città che durante la Guerra di Chioggia s'era alleata ai nemici di Venezia.

Pur non potendosi ipotizzare una conoscenza diretta, da parte del Vannozzo, del sonetto venetus, la tecnica di riproduzione del vernacolo si dimostra rispetto ad esso costante, e con esso condivide la tendenza a stabilizzare quelle forme che appaiono minoritarie nella scripta documentale coeva e nei testi non riflessi: dunque l'intera serie olto (v. 73), solto (74), Riolto (75), oltri (69), oltrier (127) per «alto», «salto», "Rialto» ecc. (si noti che in un volgarizzamento veneziano come il già citato Sent'Alban, che pure appartiene alla seconda metà del Trecento, la sola forma attestata è altro, altra, altre).$^{38} \mathrm{Idem}$ per oldire (161), oldido (45), oldì (275), loldato (299), con AU>ol, nonché anenti (68), fenti (69), danenti (99), con -an-> -en-. In Carrera, "Carrara" (19: «Sé l'arme del signor da Carera») si osserva la medesima chiusura vocalica di Ferera nel sonetto. Esiti identici anche al testo di Pignol Zucchello per quanto riguarda la labiovelare sonora: $g u->v$ - in vagnele (toscano guagnele, "vangeli»), vera ("guerra»), vardare, varenta ("guarenta»), varnazze ("guarnazze», "guarnacche»: soprabiti). Scarsamente rappresentata, nonostante la struttura dialogica del componimento, l'uscita in verbale in -s delle seconde persone singolari: a differenza del sonetto si offre solo nella cristallizzata

37. Si decurta di minime porzioni la cit. edizione del Morozzo Della Rocca, rispettandone la grafia ancorché poco coerente con le successive soluzioni dello STUSSI, Testi veneziani, cit.

38. Cfr. "Legenda de misier Sento Alban", cit., p. 45. 
(e unica) forma interrogativa, distu (84, "Co' distu», "come dici?»). Maggiore interesse offre il lessico, spesso legato alla quotidianità e destinato talvolta a sopravvivere fino a oggi : vignali (8: «vigne»), panada (25: «minestra di pane»), gotto (63: «bicchiere»; gotti anche a v. 80), tresso (v. 103, 181: «traverso", in costrutti avverbiali, «avere» e "guardare di t.»). Infine, non è forse privo di significato che l'incipit presenti un imprecazione blasfema («a le vagnele») vicina alla testimonianza dantesca, e che ancor più prossima interiezione («Sì, a le plaghe de Dio») si abbia anche nel corpo del componimento (71).

A differenza di altri testi fin qui proposti, la frottola del Vannozzo ha tradizione plurima: per quanto detto sinora e nell'esempio che si fornisce (v. 1 sg.) si segue l'edizione del Corsi, ${ }^{39}$ che si imposta su di un codice padovano della fine del Trecento (Padova, Biblioteca del Seminario, 59) emendandolo con il Laurenziano Conventi Soppressi, SS. Annunziata, 122, della prima metà del XV sec. Il testo concorda sostanzialmente con quello già fornito dal Medin nella sua edizione complessiva delle rime del Vannozzo; ${ }^{40}$ merita segnalazione che, dall'apparato del Medin, le varianti del cod. laurenziano offrano più larghi elementi di venezianità. Sistematico o quasi, ad es., il dileguo delle dentali sorde intervocaliche, secondo una tendenza «ingenua» riscontrabile nei documenti duecenteschi sopra ricordati: Laurenziano: pecchao, (sier) Storlao, (sier zan) Sanuo (Padova, seminario 59: Storlado, Sanudo), vegnuo, drio (Padova: driedo ), zittae beneeta (Padova: terra benedetta), ecc.; e anche nella lezione quel verzo abissado, il verzo del Laurenziano concorda più con il sonetto della tenzone che con il testo del cod. padovano (quel tristo abissado).

— Se Die m'aide, a le vagnele, compar,

a dir ciò che me par,

i'è gran paura:

se 'l no 'nde vien ventura,

nu perderemo l'ambladura

e difaremo foza.

Zenovesi sta in Cloza:

entro per quei vignali

li fase tutti i mali;

lo sé un peccado!

Marco Storlado

End'è pur vignudo

E sier Zan Sanudo con esso,

e enghe stadi appresso a un trar di piera:

li ha vezudo una bandiera granda

con una blanca banda

e non se che vermeio.

39. Rimatori del Trecento, a cura di G. CORSI, Torino: Utet, 1969, p. 480-495.

40. Le rime di Francesco di Vannozzo, a cura di A. Medin, Bologna: Commissione per i Testi di Lingua, 1928, p. 100-114. 
Per Sen Baseio, se la rende splendor!

Sé l'arme del signor de Carrera

Ch'end'ha fatto sta vera

Con so traditi enganni.

El fu già pluxor anni

Che sé questa bugada:

esso sì l'ha menada

e mo 'nde dà panada senza pan

- Disé mo pur plan;

Per Sen casian,

driedo ancuo' vien doman:

lassé pur andar.

Mo diseme, compar,

ch'ende può li far?

I'nde può rubar.-

Mo a che partido?

Non seremo nu a Lido?

No xè infortido 'l porto?

Mo xè Sen Marco morto?

Vu se' gran desconforto a la citade:

che Dio ve dia'nfirmitade,

lengua maledetta.

Lighéve meio la vetta,

e tollém zò la beretta

dié andar a saccomanno.

Che s'un garzon d'un anno

V'avesse oldido,

vu saresse schernido

e vituperado [...].

2.5 .

Raggiungiamo finalmente il Quattrocento con il fiorentino Burchiello, la cui musa bizzarra ricorre più d'una volta anche alla parodia dialettale. Sonetto del Burchiello quand'era a Vinegia e Sonetto di Burchiello alla veneziana è la rubrica che due manoscritti, fra i molti della tradizione, attribuiscono a un testo costruito per intero di tessere veneziane. ${ }^{41}$ Di mosaico, infatti, si tratta, più che di organica simulazione linguistica, e non solo per via della accozzaglia incoerente di termini che è tipica della produzione 'alla burchia'. Piuttosto, l'autore dimostra di non aver saputo controllare il sostrato d'origine, che riaffiora inospitamente a mettere in crisi l'impianto stesso dell'imitazione: versi come «un grosso gli vendien quella della vin», o «e negotta si bagna stando in molle» finiscono per avere, di veneziano, soltanto una voce ciascuno (vin con caduta di -o, e negotta per «nulla»: fenomeno e termine, fra l'altro, di larga dif- 
fusione in tutto il settentrione d'Italia), mentre nel contesto può stare il puro fiorentinismo vendien ("vendevano»), in buona compagnia con i vari gli, di, diesi, si (i documenti veneziani preferiscono ge, de, diese, se) e ched (con consonante eufonica) degli altri versi. La scarsità cromatica della tavolozza dialettale comporta che l'autore si dovesse attenere più rigidamente a pochi colori di base; ed è allora interessante verificare come detti colori siano in definitiva gli stessi che campivano gli esempi precedenti, a sanzionare una stereotipia della lingua lagunare ormai abbastanza stabile. Si ripropongono dunque, anche nella sede privilegiata della rima, $a l>o l$ con coldo (4) e (vero stendardo di venezianità) Riolto (4), nonché la caduta di dentale in buel (5: «budello»), vïel (11: $<$ vedel, con chiusura della protonica in iato secondario, ${ }^{42}$ "vitello»), miolle (12: "midolla», qui per "molliche»). Di notevole interesse sono i dittonghi in vocale tonica - siei (1: «sei»), miedisi (9: «medici»), zievoli (11: «cefali»), grieve (12: "gravi», "pesanti») — , che da un lato contraddicono, per frequenza, le sporadiche emersioni precedenti ${ }^{43}$ (ma si noti che il sonetto Venetus summenzionato ha struolego, e che il medesimo termine - struoligo, per la verità - offre la frottola del Vannozzo, v. 155; il veneziano odierno dice strolego), dall'altro rientrano perfettamente nello sviluppo che, come ha mostrato la Sattin, il fenomeno conobbe giusto nel Quattrocento. ${ }^{44}$

Demo a Viniesa siei capuzzi al soldo

un boccal d'acqua per un bagatttin, un grosso gli vendien quella del vin, perzò ched'el zè d'ogni tempo coldo;

un buel di tri braza di biroldo che val diesi dinari o un soldin e nu l'avemo masie da matin perzò ch'el va per Riolto il manioldo.

I miedisi han ducati per condutta e da Mestri che vien ai e zivolle e zievoli e 'l vïel che se [ne] butta.

El pane ha dure e grieve le miolle e 'mollisin e la suo crosta tutta e negotta si bagna stando in molle.

Odi contrarietà di gente folle: Vinegia è in acqua, come voi sapete, e non che loro, e can muoion di sete.

2.6.

A conclusione di questa rassegna si propone un campione nemmeno più relativo al dialetto nella sua generalità, quanto invece pertinente ad una espres-

42. Fenomeno attestato nei documenti quattrocenteschi studiati da A. SATTIN, «Ricerche sul veneziano del sec. XV (con edizione di testi)», L'Italia Dialettale, n. 49, 1986, p. 1-159, p. 68.

43. Cfr. STUSSI, Testi veneziani, cit., p. XXXIX s.

44. Cfr. SATTIN, "Ricerche sul veneziano del secolo XV», cit., p. 62-65. 
sione individuale di esso, a un vero e proprio idioletto. Tale infatti deve - o, per meglio dire, dovrebbe - considerarsi quello che Gentile Becchi, fiduciario di Lorenzo de' Medici presso la Santa Sede, raccoglie dalla viva voce di papa Paolo II, il veneziano Pietro Barbo, e verbalizza in una lettera diplomatica al Magnifico, in data 1 marzo $1471 .{ }^{45}$ Preoccupato di offrire con puntualità le parole del pontefice a chi, miglior politico di lui, ne avrebbe saputo cogliere sfumature e sottintesi («Io dissi contulisse me omnia in corde, et scrivervi questo ultimo suo precepto, acenando degli altri ragionamenti, che non ho facto nulla, ma scriptovi tutto per lasciare interpretare a voi. [...] Se io mi sentissi meglio in gambe, di giudicio o pratica, non vi straccherei in scrivervi ogni parolina, ma meglio è fatica che errore»), ${ }^{46}$ il Becchi tenta di rendere le risposte avute dal papa tal quali le ha udite: ed esse - per ciò che il testo stesso permette di giudicare - furono pronunciate in un veneziano parzialmente corretto con elementi di coiné.

Ad onta del registro palesemente colloquiale (che si coglie, ad es., nella negazione ripetuta circolarmente, "Non stemo perzò chosì male chol Re, no»), non si tratta, con ogni probabilità, di una trascrizione fedele. Anche a trascurare le illazioni sulle effettive capacità mnemoniche del Becchi risultano soprattutto sospetti alcuni elementi di difficile localizzazione, probabilmente da ricondurre all'eccessivo zelo caratterizzante dell'estensore. Complessivamente bene, dunque - ancorché generalizzabili a quel volgare "comune» che caratterizza l'Italia settentrionale del Quattrocento - ze ("ci»), Zentile, zettava, zò, zerto ecc., rasone; più decisamente veneziani meior, voia, mèia, tòiono ("migliore», "voglia», "miglia», "togliono») con passaggio -LJ- > j, ${ }^{47}$ imbasadore (il Becchi di suo scrive imbasciadore), bem, som («sono»), entenziom (con passaggio di $-n$ a $-m),{ }^{48}$ le forme verbali fasemo, zè, tegnì (imp.) e l'interrogativa con enclisi del pronome personale havivu. Sembrano invece eccessivi par per pare ("padre»), amizizia, uzèi (ci si aspetterebbe oxelli: ${ }^{49}$ "uccelli»).

Ma con tutto ciò, e appunto per il fatto di essere, prima che meccanica riproduzione, rappresentazione artificiosa di una lingua altrui, questo testo bene si colloca nella nostra serie, sottintendendo una percezione dell'idioma lagunare fatta di dati convenzionali e, insomma, di tratti dotati di qualche regolarità.

45. Il testo è stato rinvenuto e pubblicato da R. FUBINI, «Gentile Becchi tra servizio mediceo e aspirazioni cardinalizie, e una sua intervista bilingue a Papa Paolo II (1 marzo 1471)», ora in ID., Quattrocento fiorentino. Politica diplomazia cultura, Pisa: Pacini, 1996, p. 333-354 (l'edizione alle p. 350-354). Si segue qui la lezione fornita dal Fubini, diminuita di quasi tutte le parti che, nel dialogo, spettano al Becchi (ma si lascia, a titolo esemplificativo della varietà linguistica, un breve segmento introduttivo) e con minimi ritocchi grafici; al saggio di Fubini si rinvia per il contesto storico della lettera.

46. Nella cit. ed. del Fubini, p. 354.

47. Per il quale Cfr. SATTIN, «Ricerche sul veneziano del secolo XV», cit., p. 86.

48. Relativamente raro nelle più antiche attestazioni del veneziano (cfr. STUSSI, Testi veneziani, cit., p. LIX), se ne incontrano esempi epigrafici in ambito ormai cinquecentesco: "per la iuridiciom di barbacani» ancora si legge su mensola in pietra (destinata a regolare la costruzione di sporti murari, i barbacani appunto) nella realtina Calle de la Madonna, a Venezia.

49. Forme dei documenti editi da Stussi: cfr. Testi veneziani, cit., p. 236. 
[...] «Lorenzo, Beatissime Pater, si raccomanda humilmente a' piedi di Vostra Beatitudine. Ha intese le parole amorevoli et benigne che Vostra Sanctità ha di lui usate con questo imbasciadore ducale; sa nisuna lettera o testificazione potere exprimere l'animo suo; pure me ha adiritta questa, et commesso quello ch'io posso, io faccia a boccha; il resto spera che la experientia et conditione de' presenti tempi dimosterrà meglio». Lesse la lettera, poi rispuse: «Nui l'havemo dicto adesso a questo imbasadore: Lorenzo ze par meior che so par, più discreto et più moderato; Piero se zettava troppo et alterava». Rispusi: «La Sanctità Vostra suole predire le cose; non so se ha perduta la sua divinatione, che giudica hora sì da presso et chosa sì chiara». Rise et seguì: «Pure me ha con lo imbasadore venetiano che è là morso a questi zorni, che contribuisco poco et lento [...].» Disse: «E' credo bem che 'l sia vero, che fasemo più che non possiamo. Messer Zentile, nui v'havemo dicto altra fiada che per Fiorentini non zè più conforme né più stabile amicitia che questa della Chiesa. Tutte le altre, et sia chi se voia, sono meno durabile et più rapaze. Vu dirì: «O la Chiesa per la morte dei pontefici non varia ella?». Digho che nella terra de' Zieghi Argo è chi z'ha uno ochio. [...] Hor a proposito più stabile è questa che nisuna altra amizizia, et non è rapase, chome siria di Vinesia, che benché durabile, è perzò mala compagna. Et chosì quest'altri tutti voriano deventare più grandi. Som uzèi de rapto». [...] "La Chiesa — disìmi - confina choi Fiorentini più de 40 mèia; chome e' disse adesso al vostro embasciadore, ha'lla mai tolto niente del vostro? Non havivu più presto del so?». [...] Riprese: «non disì chosì. Più z'ha inzurià il Duca che 'l Re. Se 'l se dimostrò più a Rimino, tanto pecca chi tiene quanto chi che scortica, et la prohibitiom dise consilium et consensum, [...]. Ma quando ze volavamo collegare chol Re et Venitiani, non ze pregò il Duca per Nicodemo del contrario, dandoze entenziom, et po' per sé medesimo senza nostra saputa fe' la lega particulare, et vu le prozessioni, con molti capitoli de diretto contro di nui? Non stemo perzò chosì male chol Re, no. La rasone, il tempo, la experientia lo ha bene aconzo [...].

\section{3.}

L'intera storia del veneziano scritto è attraversata da abitudini grafiche di sostanziale appoggio a quelle della lingua, che si definiscono già nel XVI secolo e si mantengono sostanzialmente operative per un lungo arco secolare. Non è questa la sede per offrire, nemmeno in termini elementari, un panorama di una tradizione letteraria straordinariamente ricca, che si definisce tanto nella pratica della scrittura di un veneziano civil - centrale, cittadino e dominante quanto nel rigoglio connotativo ed espressivo delle sue varianti, con insistenza sui caratteri di perifericità ed arcaicità. La precoce registrazione quattrocentesca del siciliano Caio Caloiro Ponzio nota già la desinenza pronominale in ào (portao, donao, contro alla riduzione di portà, donà ecc. del centro) come «spiacevole» e caratteristica dei quartieri periferici. Non è un caso che proprio questa marca diventi il carattere privilegiato della figura del vecchio veneziano da commedia, che si fossilizza poi nella maschera di Pantalone. Se tra Quattrocento e Cinquecento il veneziano tocca tutti i generi — ascendendo dalla liri- 
ca di intonazione popolaresca al canzoniere organico, fissando altresì la tradizione di una prosa epistolare - sono i fasti del secolo della «letteratura dialettale riflessa", in operazioni di grande cimento (si pensi alla traduzione dei "primi poemi», come per esempio La Gerusalemme liberata), a testimoniare la ricchezza inesauribile e la possibilità di infinita ricezione delle «ricche miniere» del veneziano.

Una pagina di grande interesse è costituita dalla prefazione che Carlo Goldoni scrive per i suoi Rusteghi, andati in scena nel carnevale 1760 e pubblicati nel terzo tomo dell'edizione Pasquali (1762), dove l'annotazione certo occasionale riflette una svolta epocale. ${ }^{50}$ Goldoni torna qui, anzitutto, a discutere la questione da lui a lungo dibattuta — fin dalla prima scelta di pubblicare le sue commedie in veneziano accanto a quelle in lingua - dell'offerta sulla pagina a un pubblico interamente italiano di lettori ciò che era stato pensato originalmente per la più ridotta offerta a una platea cittadina di spettatori. Non è qui in questione quella ricchezza e modulazione che permette la restituzione di una variata tavolozza di espressioni letterarie - che in uno spazio plurisecolare aveva anzi privilegiato inflessioni arcaiche, gergali e variamente coloristiche - quanto la pretesa che una lingua e una dimensione del quotidiano da essa espressa - quella che l'autore definisce il suo "sapore dei sentimenti» risultino comprensibili su più larga scala. La questione è stata, a questa altezza, discussa più volte da Goldoni a partire dalla prefazione a La putta onorata (nel secondo volume dell'edizione Bettinelli, 1751). Una preoccupazione che poteva, dieci anni dopo, ritenersi sostanzialmente superata dal successo e dalla diffusione della drammaturgia in dialetto accanto a quella in lingua, cementati anziché ostacolati dal passaggio dalla scena alla pagina. Ed è appunto nella prefazione ai Rusteghi — nel passaggio dalla piazza all'interno domestico e dalla lingua "atmosferica» del popolo a quella chiusa di borghesi minimi — che Goldoni spende alcune riflessioni sulla scrittura del veneziano.

Goldoni aveva già dovuto precedentemente, sul campo, farsi glossatore di sé stesso, offrendo ai lettori non veneti delle annotazioni sostanzialmente di carattere lessicale o di illuminazione fraseologica ( Ho data la spiegazione a tutti quei termini, e a quelle frasi, che non possono dagli stranieri rinvenirsi nei vocabolari italiani»), arrivando a più riprese a promettere un vero e proprio Dizionario del veneziano, o, almeno, del suo veneziano di autore. Questo strumento - per la prima volta abbozzato in forma minima nel glossario che faceva seguito alla pubblicazione postuma delle satire veneziane di Dario Varotari (Il vespaio stuzzicato, 1671) — non troverà realizzazione e l'impresa — su basi tutt'altre — sarà tentata dalla fatica tardo-settecentesca del Patriarchi e — rimasto manoscritto l'altro progetto del Muazzo ${ }^{51}$ — compiutamente realizzata dal Dizionario del Boerio. Goldoni intravede però, anche solo per

50. Il testo si cita dall'edizione a cura di Giuseppe Ortolani di Tutte le opere, Milano: Mondadori, VII, $1946\left(1973^{4}\right)$, p. 625-626.

51. Cfr. Paolo Zolli, "La "Raccolta de' proverbi, detti, sentenze, parole e frasi veneziane" di F. Z. Muazzo", in Studi Veneziani, n. 11, 1969, p. 537-582. 
un'istante, un ordine di preoccupazioni che supera il piano del lessico per entrare in quello della grammatica:

Per esempio, le coniugazioni de' verbi sono alquanto diverse, ma si capiscono facilmente: farave per farei; son andà per sono andato; se savessi in luogo di se sapeste, non sono modi sì strani che abbiano bisogno di spiegazione, né basterebbe il dizionario a spiegarli, ma vi vorrebbe ancor la grammatica.

Liquidato così lapidariamente il problema, l'ultima parte della prefazione si sofferma sulla questione forse capitale, quella di una ortografia, che emerge come evidentemente disattesa, e nonostante — oggi si può dire, dopo debiti approfondimenti filologici - la tutt'altro che occasionale applicazione di Goldoni alla propria scrittura:

Anche l'ortografia veneziana altera talvolta il significato, ma chi vi abbada l'intende, ed è l'ortografia regolata secondo il suono della pronuncia. Noi, per esempio, non diciam bello, ma belo, non perfetto, ma perfeto; e per regola generale quasi tutte le consonanti doppie da noi si pronunciano semplici. Però in alcune voci le lettere semplici da noi si raddoppiano, come in luogo di cosa noi diciam cossa, ma queste son pochissime.

È evidente che «l'ortografia regolata secondo il suono della pronuncia» resti un criterio enunciato come di riferimento ma sostanzialmente evaso nella scrittura effettiva, a partire dalla tolleranza nell'intera opera goldoniana in veneziano - a proposito del caso più semplice, quella dello scempiamento delle consonanti - tra grafie fonetiche e grafie italianeggianti, per oscillazione dell'uso da parte dello stesso autore e per indifferenza dei responsabili della composizione tipografica.

Né questa volontà - ciò che forse più sorprende - fu realizzata dagli editori goldoniani, quando, non certo mossi da criteri di conservatività a scopo di documentazione, essi desiderarono conferire una maggiore uniformità di scelte grafiche, ma imboccando decisamente la strada opposta. Il maggiore studioso goldoniano della prima metà del Novecento, Giuseppe Ortolani, realizzò infatti un'edizione dell'intera opera scegliendo infatti per l'allestimento della sua grande fatica la via - già perfettamente visibile nelle edizioni tardosettecentesche e in particolare nella prima davvero completa, la veneziana Zatta — di una prevalente riconduzione alla grafia italianeggiante, per esempio con sistematica preferenza del raddoppiamento rispetto allo scempiamento consonantico.

Nella pagina goldoniana che si è citata la riflessione linguistica viene offerta al lettore non attraverso categorizzazioni, ma tramite una breve, e tutto sommato casuale, scelta di «esempi». D'altra parte il criterio che guida l'autore di fronte a testi «da leggere» (sono del tutto vuote e retrospettive le idee che Goldoni potesse porsi il problema di una «intonazione ad alta voce» della sua lingua teatrale da parte del lettore comune, posta la separata e non discutibile competenza dei comici) non è quello della corretta dizione ma della «distinzione» dall'italiano. Dal che consegue che ciò che assomiglia all'italiano - o 
che con esso rischia, nell'indistinzione, di confondersi - conta meno di ciò che non gli assomiglia, e che quindi ha bisogno di essere chiarito. Non sono fonetica e morfologia categorie, dunque, forti in questo ordine di preoccupazioni, ma lo è semplicemente - come era sempre fin là apparso alla tradizione - il lessico. Ed il problema non muta sostanzialmente - anche se completamente diversa e per certi versi opposta è la coscienza culturale che illumina la questione — dalla percezione della cosiddetta letteratura dialettale riflessa. Quella si era definita e beata dalla complessità e particolarità, e tanto più dalla difficoltà, lateralità, espressività, di un patrimonio lessicale e fraseologico, questa si poneva l'obiettivo della chiarezza e della traducibilità: una strada nuova che - come si è brevemente visto - conduce tuttavia all'ipotesi del dizionario come strumento d'illuminazione delle "differenze» rispetto all'italiano parlato della comune conversazione, non della grammatica.

4.

Le righe dichiarative, con cui la breve Grammatica del Manin comincia, offrono, con evidenza un secondo e importante punto di osservazione, laddove la questione di una definizione grafico-fonetica, prima ancora che morfologica, definisce tale istanza dell'illuminazione della scrittura del veneziano, $\mathrm{ma}$ in una prospettiva sostanzialmente disinteressata alla letteratura e, dunque, alla sua convenzionale e secolarmente codificata serie di abitudini scrittorie. Scrive Manin:

I pochi scrittori che abbiamo nel viniziano dialetto usarono diversa ortografia e quella scelsero che più loro attalentava e più si avvicinava alla toscana. Io allo 'ncontro mi studierò di scrivere in modo che senza fatica colui che non è della veneta pronunzia perito possa dare alle parole quel suono che i viniziani stessi loro attribuiscono.

La scarsa considerazione - e forse la scarsa conoscenza del reale patrimonio letterario veneziano (è del 1832 la fondamentale ricognizione storica attraverso il catalogo bibliografico tentata dall'erudito Bartolomeo Gamba con la Serie degli scritti impressi in dialetto veneziano) - che emerge dal riferimento ai "pochi scrittori», insieme alla riduzione a una sorta di libertà di comodo di norme in realtà implicite ("quella scelsero che più loro attalentava»), mostra come il progetto prescindesse dalla volontà di una documentazione storica. Così lo stesso, assai più esatto, riferimento all'appiattimento sulla grafia del toscano letterario - tenendo anche presente ciò che Manin non sapeva o non considerava, a proposito del ruolo primario di Venezia e del Veneto nella cinquecentesca grammaticalizzazione dell'italiano - mostrava una sostanziale indifferenza alla storia della tradizione scrittoria. In una diversa prospettiva è invece proprio questo tratto - principale e caratterizzante lungo i secoli - a definire attraverso la letteratura in veneziano, e certo non pochi scrittori, la codificazione di fatto della sua scrittura. 
Possiamo citare, a titolo d'esempio, alcuni tratti che definiscono le fondamentali abitudini scrittorie, che distanziano, in un arco come si è detto secolare, la rappresentazione fonetica attraverso la riconduzione alla grafia italiana. Anzitutto la rinuncia alla rappresentazione del tipico tratto della $l$ intervocalica evanescente (solo, e saltuariamente, registrata in testi che implicano la caricatura linguistica del veneziano isolano, dove quel tratto appariva evidentemente più forte, come in un dialogo tra pescatori della seconda metà del ' 500 in cui leggiamo non già balo per ballo ma addirittura bao: $:^{52}$ appartengono, del resto, solo a una (laterale) pratica recente e alla scrittura dei semicolti alcuni tentativi per altri versi ovviamente fuorvianti, di rappresentazione fonetica - soprattutto in iscrizioni e graffiti - della $l$ evanescente intervocalica con $e$ o $i$, per esempio di solo come soeo o soio). O ancora la rappresentazione con chi + vocale tanto di chi-velare che di ci-palatale, per cui Checca o cheba risultano indistinti da chiave o chiapar (che si pronunciano ciave e ciapar), giungendo addirittura a scritture «aberranti» come ciacole > chiacole (ma curiosamente lo stesso fenomeno di attrazione della grafia italiana non si dà però sempre per $g h i$ I $g i$-, dove, per esempio, si possono incontrare — soprattutto in testi del Cinque e del Seicento - grafie come giazzo e non ghiazzo, giozza e non ghiozza ecc.).

Il cenno che abbiamo già visto in Goldoni al «raddoppiamento» di $s$ dall'italiano $\cos a$ a cossa, tocca, in realtà, per via approssimativa, l'essenziale questione della rappresentazione della sorda e della sonora nel veneziano scritto. La medesima questione che implica la messa in campo del grafema speciale $x$ per il consistente impiego in rapporto alla terza persona singolare dell'indicativo del verbo essere e il saltuario, ma comunque rilevante, utilizzo per forme del verbo dire, come dice > dixe, nell'assenza nella scrittura dei secoli XV-XVIII dell'uso distintivo della cediglia, o ancora nella tipica forma Venexia. Questione più rilevante e controversa - a cui gli ultimi esempi ci avvicinano — è quella del valore fonetico del grafema $z$ (e, di conseguenza, di $c$, non più usufruito nei secoli XVI-XVIII e introdotto a scopo modernamente, a scopo diacritico, in alcune edizioni di testi cinque-settecenteschi). Il Discorso preliminare del Boerio al suo Dizionario dichiara addirittura "...un vezzo o un mendo, contratto fin dalla fanciulezza per l'ignoranza o l'inavvertenza di chi insegna a parlare..." («non solo la plebe veneziana, ma molte altre persone») la pronunciare di «cc e $c i$ ed anche la $z$ aspra, come se fossero una $s$ dolce»: "Dicono per esempio sinque per cinque, sinquesento per cinquecento, seola per ceola...", ecc. E questa testimonianza ha fondato, addirittura, una supposta pronuncia arcaica rispetto a quella del veneziano odierno (che sarebbe, così, da intendersi quella della "corruzione» plebea indicata da Boerio). Mentre tale alternanza è - oggi come nella memoria storica dei parlanti veneziano - quella tra un piano più genuinamente dialettale e l'innalzamento verso una fonetica appoggiata all'italiano, bisognerebbe pure fare qualche debita tara a una simile affermazione. Considerare, dunque, con la dovuta attenzione, da una parte, la scarsa credibilità storica dell'imputazione a un fenomeno corruttivo per malcostu- 
me linguistico di chi insegna. Com'è sostenibile, infatti, l'idea che il popolo, o "la plebe veneziana" possa essere stata traviata in un uso diffuso - e Boerio dice di più: pressoché generalizzato - come chi apprendesse da un cattivo maestro una lingua straniera? Dall'altra osservare lo scarso credito della prova "storica» addotta per la pronuncia ritenuta corretta, che è, appunto, un rinvio al sistema grafico anfibio della pratica scritta del veneziano: «Non troverassi alcun autore veneziano antico o moderno, comunque egli stesso così parlasse, che siasi pensato di scrivere in cotal guisa...", come se il sistema grafico - poniamo - di Carlo Goldoni non riflettesse semplicemente le abitudini scrittorie dell' italiano, comunque, appunto, Goldoni parlasse.

Questi problemi si rendono meglio evidenti se si passa, per esempio, in rapporto a Goldoni (per il quale non disponiamo peraltro di autografi) alle abitudini grafiche del maggiore scrittore di teatro veneziano dell'Ottocento, Giacinto Gallina, in un'epoca in cui agiscono più visibilmente le spinte opposte della registrazione del «colore» dialettale — spesso con eccesso di pittoresco - e di una più forte istanza di grammaticalizzazione. Il sistema di scrittura di Gallina - anche a partire dalla possibilità di confronto dei testi a stampa con i manoscritti - presenta una casistica più ampia ma anche evidenti segni dell'imporsi di un grado maggiore di «resa grafica della pronuncia» rispetto al modello goldoniano. ${ }^{53}$

L'alternanza tra forme "fonetiche» e forme "grafiche» offre certo un'indicazione preziosa in questo senso, nelle oscillazioni tra forme quali sucaro e zucaro, sate e zate, sigo e zigo, suca e zuca, ecc. e, quindi, per esempio, insucar a inzucar. Ciò tenuto conto che l'uso di $z$ semplice ha significato davvero distintivo, di indicazione almeno sommariamente fonetica, nel veneziano laddove essa segnala - diverso naturalmente da $z$ italiana - l'esito J + vocale, e dunque zogo ("gioco»), zogia ("gioia»), za ("già»). All'interno di parola la pronuncia veneta $-a z o,-a z a$ e simili produce (anche nei manoscritti autografi) l'alternanza di grafie «fonetiche» con ss e di grafie stereotipa con $z$, o addirittura con $z z$, procedenti, la prima, dalla grafia italiana venetizzata coll'indicazione di scempia e di sibilante sorda semplice (per esempio palasso e palazo, piaza e piassa) e la seconda da mera sovrapposizione dell'abitudine grafica italiana.

L'oscillazione nel sistema di Gallina offre non già la prova dell'emergere, proprio allora, del malcostume plebeo - o della sua registrazione saltuaria e indifferente in commedia - quanto, piuttosto, l'incrinarsi di un sistema grafico convenzionale, proprio in virtù di una doppia, parallela, influenza di quelle istanze di riforma ortografica così vigorosamente respinte dal lessicografo Boerio: «Ho sentito qualche zelante dell'ortografia ad opinare che così dunque si dovesse scrivere come la maggior parte pronuncia». L'unico movente davvero fondato e davvero ragionevole delle scuse di Boerio restava - e resta — quello di non «alterare anzi capovolgere l'ordine alfabetico di migliaia di voci». 
Ora è proprio su questo punto - parallelamente all'emergere delle grafie che preferiscono quantitativamente $s$ a $z$ nella registrazione del parlato galliniana - che interviene la prova fornita dalla Grammatica di Daniele Manin, che sembra appunto contraddire il criterio che abbiamo sospettato mosso da preoccupazioni di lessicografo del peraltro non veneziano Boerio:

La $c$ colla virgoletta ha il suono di un $s$ aspro o vogliam dire del $c$ francese innanzi alle vocali $e, i$ : p. e. çénto, cento, çémbalo, gravicembalo.

$[\ldots]$

$\mathrm{Li}$ viniziani non fanno mai sentire alcuna consonante doppia, appoggiandovi fortemente la voce: laonde riesce superfluo allo scrittore lo addoppiarle; eccettuata soltanto la $s$ che in tal caso, non acquistando peraltro alcuna vibratezza, assume il suono di una s forte o vogliamo dire d'un sigma greco.

Questa $s$ addoppiata si usa nelle voci che scrivendo italianamente dovrebbero avere una $s$ doppia od un zeta aspro: p. e. adèsso, adesso, aflissión, afflizione, Venessia, Venezia. 